\section{Adaptation due to symbionts and conflicts between heritable agents of biological information}

\author{
Simon Fellous, Olivier Duron and François Rousset
}

Calls for an extended evolutionary synthesis are flourishing in the scientific literature (as highlighted by a recent Review article in this journal (Beyond DNA: integrating exclusive inheritance into an extended theory of evolution. Nature Reviews Genetics 12, 475-486 $\left.(2011)^{1}\right)$ and REFS 2,3), so the identification of the necessary 'extensions' has become a crucial goal to many evolutionary biologists. Here we wish to emphasize two elements: the participation of symbionts in their hosts' adaptation and the potential for evolutionary conflict between the different agents of biological information that affect the phenotype of an individual, including symbionts.

In insects, bacterial symbionts can improve host fitness, and recent studies have shown that these symbionts respond to the selection on their host's phenotype by increasing in frequency, hence permitting the adaptation of the host ${ }^{4-8}$. For example, within a few years, a strain of a maternally transmitted bacterium belonging to the Spiroplasma genus has invaded many North American populations of its host, Drosophila neotestacea, because of the protection it provides against a parasitic nematode ${ }^{6}$. The host has thus become resistant to the parasite owing to the presence of the symbiont, which is now part of the host's extended genome.

Beneficial, vertically transmitted symbionts can be found in numerous taxa. In addition to the well-described interactions between insects and their bacterial symbionts (see above), the adaptation of host populations based on the spread of beneficial symbionts has also been shown in plants that are defended by symbiotic fungi ${ }^{9}$. Similar processes probably also involve viruses
- which can be beneficial to plant, bacterial, vertebrate and invertebrate hosts ${ }^{10}$ - and the bacterial communities living on and in vertebrates such as humans (for example, REF. 11). The strongest evidence for the importance of symbionts as sources of evolutionary innovation probably remains the great prevalence of mitochondria and chloroplasts in eukaryotes and the symbiotic origin of these organelles $^{12}$.

The presence within the same organism of different sources of biological information (for example, nuclear genes and symbionts) that have different effects on the phenotype and different modes of transmission may also lead to evolutionary conflicts rather than favouring adaptation. Again, such conflicts are well-described in insect-bacterium associations. Indeed, several bacteria that have recently been shown to be involved in host adaptation were previously known as parasitic manipulators of sex determinism and reproduction (for example, REFS 5,6). Conflicts may even occur when components of inclusive inheritance are transmitted in the same manner. For example, Danchin et al. discuss the differential imprinting of maternally and paternally derived genes ${ }^{1}$ as a component of inclusive inheritance. Actually, conflicts between nuclear genes controlling imprinting in both parents and the offspring are the leading hypothesis for the evolutionary origin of such imprinting ${ }^{13,14}$. An inclusive understanding of the different types of biological information and their influence on phenotypic evolution thus necessitates the simultaneous consideration of these different elements and their potential conflicts.
Simon Fellous, Olivier Duron and François Rousset are at the Institute of Evolutionary Sciences - Montpellier, UMR 5554 ISE-M, University of Montpellier 2, CNRS,

Place Eugene Bataillon, 34095 Montpellier, France. e-mails: simonfellous@free.fr; olivier.duron@univ-montp2.fr; Francois.Rousset@univ-montp2.fr doi: 10.1038/nrg3036-c1 Published online 18 August 2011

1. Danchin, E. et al. Beyond DNA: integrating inclusive inheritance into an extended theory of evolution. Nature Rev. Genet. 12, 475-486 (2011).

2. Jablonka, E. \& Lamb, M. J. Evolution in Four Dimensions: Genetic, Epigenetic, Behavioral, and Symbolic Variation in the History of Life (MIT Press, Cambridge, Massachusetts, 2005).

3. Pigliucci, M. Do we need an extended evolutionary synthesis? Evolution 61, 2743-2749 (2007)

Dunbar, H. E., Wilson, A. C. C., Ferguson, N. R. \& Moran, N. A. Aphid thermal tolerance is governed by a point mutation in bacterial symbionts. PLOS Biol. 5, e96 (2007).

5. Himler, A. G. et al. Rapid spread of a bacterial symbiont in an invasive whitefly is driven by fitness benefits and female bias. Science 332, 254-256 (2011).

6. Jaenike, J., Unckless, R., Cockburn, S. N., Boelio, L. M. \& Perlman, S. J. Adaptation via symbiosis: recent spread of a Drosophila defensive symbiont. Science 329, 212-215 (2010).

7. Oliver, K. M., Campos, J., Moran, N. A. \& Hunter, M. S. Population dynamics of defensive symbionts in aphids. Proc. R. Soc. B. 275, 293-299 (2008).

8. Weeks, A. R., Turelli, M., Harcombe, W. R., Reynolds, K. T. \& Hoffmann, A. A. From parasite to mutualist: rapid evolution of Wolbachia in natural populations of Drosophila. PLoS Biol. 5, e 114 (2007).

9. Clay, K., Holah, J. \& Rudgers, J. A. Herbivores cause a rapid increase in hereditary symbiosis and alter plant community composition. Proc. Natl Acad. Sci. USA. 102, 12465-12470 (2005)

10. Roossinck, M. J. The good viruses: viral mutualistic symbioses. Nature Reviews Microbiol. 9, 99-108 (2011).

11. Costello, E. K. et al. Bacterial community variation in human body habitats across space and time. Science 326, 1694-1697 (2009).

12. Margulis, L. \& Fester, R. Symbiosis as a Source of Evolutionary Innovation: Speciation and Morphogenesis (MIT Press, Cambridge, Massachusetts, 1991).

13. Burt, A. $\&$ Trivers, R. Genetic conflicts in genomic imprinting. Proc. R. Soc. Lond. B. 265, 2393-2397 (1998).

14. Brandvain, Y., Van Cleve, J., Ubeda, F. \& Wilkins, J. F. Demography, kinship, and the evolving theory of genomic imprinting. Trends Genet. 27, 251-257 (2011).

Acknowledgements

S.F. was supported by the French Agence Nationale de la Recherche (grants ANR-09-BLAN-0099 and ANR-09PEXT-011).

Competing interests statement

The authors declare no competing financial interests.

\section{FURTHER INFORMATION}

Simon Fellous's homepage:

http://simonfellous.free.fr/research/gate.htm 